\title{
Hotaru: Intuitive Manipulation Techniques for Projected Displays of Mobile Devices
}

\author{
Masanori Sugimoto, Kosuke Miyahara, Hiroshi Inoue, and Yuji Tsunesada \\ University of Tokyo, Graduate School of Frontier Informatics, \\ 5-1-5 Kashiwanoha, Kashiwa, Chiba, 277-8561, Japan \\ \{sugi, miyahara, inoue, tsunesada\}@itl.t.u-tokyo.ac.jp \\ http://www.itl.t.u-tokyo.ac.jp/
}

\begin{abstract}
Mobile devices (cellular phone, PDA, etc.) have so far been personal tools. Due to their evolution to multi-functionality, however, the devices have begun to be used by multiple people in co-located situations. This paper discusses near future technologies: a mobile device with a projector and intuitive manipulation techniques by using a video camera mounted on the device. In today's technologies, it is difficult to realize a mobile device with a small and lightweight projector that still retains the feature of mobility. Therefore, we have developed a system to project displays of mobile devices on a table, a floor, or a wall, by tracking their three-dimensional positions and orientations and using an existing LCD projector. The proposed system called Hotaru (a firefly, in English) allows users to annotate/rotate a picture or a document in a mobile device by touching its projected display with their fingers. Users can intuitively transfer a file between multiple devices by making their projected displays overlapped. Informal evaluations of Hotaru indicated that the proposed manipulation techniques could effectively support multiple people in co-located situations in conducting their tasks.
\end{abstract}

\section{Introduction}

Mobile devices (PDA, cellular phone etc.) have rapidly penetrated into our society and many people use them in their daily lives. For example, in Japan in 2003, the number of subscribers of cellular phones has amounted to 86 million, which is about three fourths of Japanese total population [4. One of the recent trends of cellular phones is multi-functional: not only a phone to communicate with a person in a remote location but also a web browser, a digital video camera, a game machine, a music player, a television, a GPS, and so on. Although the growing trend toward more functions has been remarkably observed in cellular phones, the other types of mobile devices also exhibit the similar tendencies. For instance, various commercial accessories attachable to PDAs for extending their functions are available. This trend makes the differences between a mobile device and a personal computer smaller: a cellular phone or a PDA is becoming a computer that has almost the same functionality of desktop/laptop computers retaining the feature of mobility. Actually, mobile devices have been used as a 
personal tool such as a personal scheduling assistant, and recently have begun to be used by multiple people in face-to-face or co-located situations.

Let us show you some examples. When you take a photo by using a digital camera mounted on your cellular phone, you may want to show the photo to (more than two) people around you. However, due to a problem of screen real estate of a cellular phone, it is not easy for multiple people to simultaneously look at the photo on your cellular phone. Moreover, when you send the photo to a person who has requested it, you need to conduct unintuitive and bothersome operations on your phone (e.g. search his mail address, attach the photo to a mail, and send it through the user interface of the phone).

Suppose that the display of your cellular phone can be projected on a wall in front of you. If your phone that mounts a projector is as lightweight and small as the recent models of cellular phones, thanks to its mobility, you can make the projected display appear anywhere on a wall, a ceiling, a floor, or a table. Therefore, you can easily look at a photo taken through your cellular phone with people around you. As your cellular phone is equipped with a digital video camera, it may also be possible to capture an image of its projected display. If the cellular phone can recognize manipulations conducted with fingers on its projected display, for example, selecting a file or starting an application program, it will be able to effectively support conversations or collaborative tasks of multiple people in co-located situations. As users can freely change the projected locations of the displays by moving their own cellular phones, the users can conduct data transfer between their phones by overlapping their projected displays.

We believe that a mobile device that mounts a video camera and a projector has the possibility to provide us with a new technique for supporting co-located collaboration in a ubiquitous computing environment. Canesta Keyboard [9] is an one-color projection system designed to be attached to a PDA and used as a personal virtual keyboard (therefore, inappropriate as a shared display for multiple people). Unfortunately, a full-color projector mountable on a mobile device is currently not available due to its weight and power consumption. However, according to the recent news, several researches on portable projectors are in progress and those mountable on mobile devices will become available in near future [5]. In this paper, therefore, we propose a system called Hotaru (a firefly in English) that allows users to conduct intuitive manipulations on projected displays of mobile devices by utilizing currently available technologies.

The paper is organized as follows: Section 2 shows the related work to Hotaru. In Section 3, the system configuration of Hotaru is described. Section 4 shows several manipulation techniques by using Hotaru. Section 5 discusses the user studies of Hotaru. Finally, Section 6 concludes the paper.

\section{Related Work}

Hotaru is related to several research topics such as mobile and ubiquitous computing, finger recognition, manipulation techniques for projected displays, and so on. There are too many predecessors related to Hotaru. Therefore, some of them 
which seem similar to and should be differentiated from Hotaru are discussed in this section.

iLamps [7] provides users with adaptive projection techniques with a cluster of handheld projectors. iLamps identifies locations and orientations of multiple projectors and creates a seamless and consistent image over a projected surface (e.g. planar, spherical etc.) by correcting an overlapping image given by the projectors. The main purpose of iLamps is different from that of Hotaru in that it proposes manipulation techniques for overlapping projected displays of mobile devices.

In [6], the concept of "steerable interface" and its implementation are described. The proposed system (ED: Everywhere Display) uses an LCD projector fixed to a ceiling and can project a display on any surface (e.g. a wall or a floor in a room) by tracking a user's head position and controlling an angle of a mirror attached to the projector. Therefore, ED does not allow multiple users to freely change the locations, shapes, and sizes of their projected displays as the users like, which is possible for users of Hotaru.

HyperPalette [1] allows a user to conduct intuitive operations with his PDA. A user can bring a photo projected on a table into his PDA (scoop), or drop a photo in the PDA onto the table (spread) by tilting and moving the PDA. The difference between HyperPalette and Hotaru is that Hotaru allows a person without a PDA to join collaborative tasks by annotating on a projected display with his/her finger. Moreover, Hotaru provides users a more intuitive and direct method by utilizing overlapping projected displays of multiple mobile devices, although HyperPalette proposes a data transfer method between multiple PDAs via a table on which the data to be transferred is projected.

Augmented Surfaces [8] allows users to drag files, such as documents or images, to be shown on a computer desktop, a table or a wall, by using a laser pointer. Users can easily share these files with other users and bring them into their own personal computers. The difference between Augmented Surfaces and Hotaru is that Hotaru provides users with intuitive methods for supporting their collaborative tasks such as annotating by the users' fingers.

In [2], a system that visualizes a personal and a public spaces on a screen of each user's PDA is described. A user can place a file in the public space to make it visible and accessible to all users, or bring a file from the public space to his personal space. This system does not allow users to specify who can access to which files in an intuitive manner. On the other hand, a user of Hotaru can select a person (or PDA) and a file by overlapping their projected displays in an intuitive manner.

\section{System Configuration of Hotaru}

\subsection{Design and Implementation Overview}

In order to realize projected displays of mobile devices by using existing LCD projectors (most of them weigh more than $1 \mathrm{~kg}$ ), the following requirements must be satisfied: 
1. Three-dimensional (3D) positions and orientations of mobile devices are automatically identified.

2. Based on their 3D positions and orientations, the locations, sizes, and shapes of their projected displays are automatically determined.

In order to fully satisfy the requirement 2, a special apparatus to control the positions and orientations of LCD projectors will be necessary. However, it is almost impossible to implement such an apparatus that can instantaneously change their positions and orientations by following 3D moves of multiple mobile devices. On the other hand, it is possible to investigate the proposed idea by partially (not completely) realizing the feature of projected displays of mobile devices. Therefore, we decided to develop a prototype version of Hotaru as shown in Fig. 11. In this figure, a stereo camera is installed above users in order to identify 3D positions and locations of their mobile devices. Based on their 3D positions and locations, the sizes and shapes of the screen images of the devices are calculated and projected onto a table or a wall through an LCD projector.

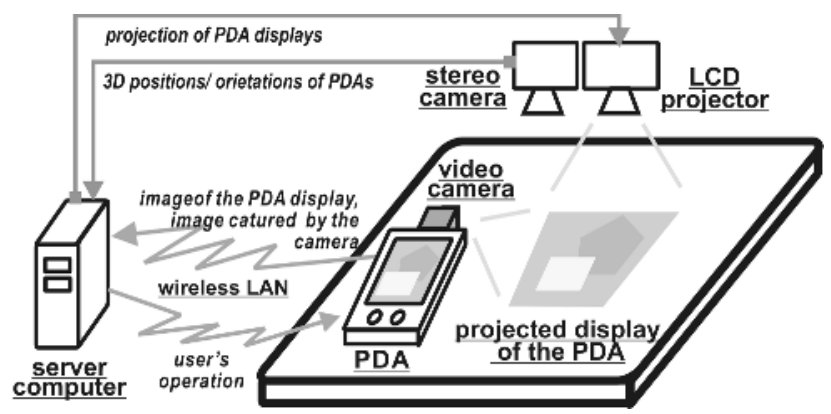

Fig. 1. System configuration of Hotaru

In order to capture images of projected displays where users' manipulations are conducted, a video camera attached to a mobile device is used. In the prototype version of Hotaru, we use a PDA as a mobile device, because cellular phone vendors do not fully release the technical specification of their devices. Due to the limited computational capability of current models of PDAs, an image processing module for recognizing users' manipulations on projected displays is executed on the server computer.

The current version of Hotaru imposes one restriction on its users: displays of users' PDAs are projected only within a specified area (e.g. on a table or a wall) determined by the installation positions and angles of the stereo camera and LCD projector. However, the other functions, such as recognizing a user's manipulation on a projected display through a camera mounted on his PDA, are fully realized. Therefore, when a projector mountable on a mobile device has become available, the techniques described in this paper will also be available without any modification. 


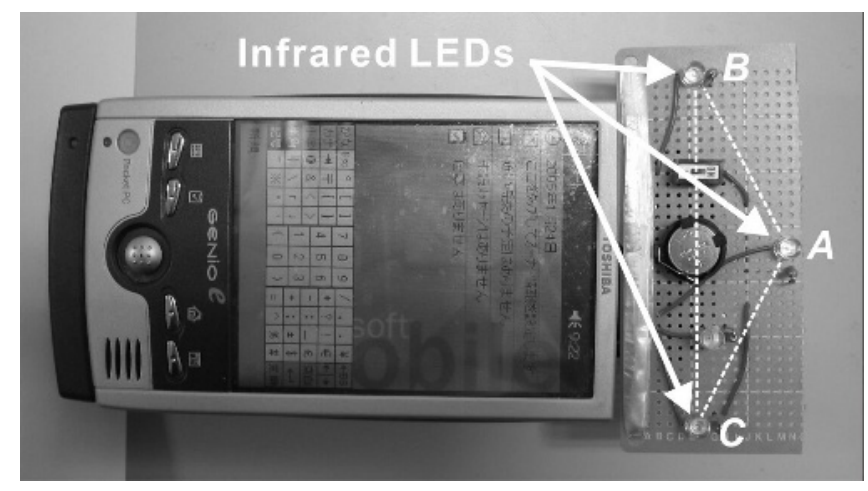

Fig. 2. Infrared LEDs for position and orientation identification

\subsection{Location and Orientation Identification}

A marker with infrared (IR) LEDs and a stereo camera are used for recognizing the 3D position and orientation of a user's PDA. The IR LEDs on a circuit board are arranged to form an isosceles triangle $(A, B$, and $C$ in Fig. 2). The position of the PDA is represented as $P=\left(x_{p}, y_{p}, z_{p}\right)$ which is the coordinates of the centroid of the triangle. The orientation of the PDA is calculated by the sum of the vector $\overrightarrow{B A}$ and $\overrightarrow{C A}$, and represented as $\mathbf{d}=\left(x_{o}, y_{o}, z_{o}\right)$. Different blinking patterns are assigned to LEDs at the vertex $A$ for identifying individual PDAs.

Experimental results to evaluate this method have proved that the position and orientation recognition errors are less than six centimeters and ten degrees, respectively. In the current implementation, it takes less than one second to correctly recognize individual blinking patterns of LEDs. This means that a user is required to hold his PDA steadily for one second so that Hotaru can identify the PDA successfully.

\subsection{Projection of Displays}

In order to emulate a PDA that mounts a projector, the location, size, and shape of its projected display must be determined based on the position and orientation of the PDA. Let the projecting plane (the surface where a display of a PDA at the point $P$ is projected) be $\Pi$ and its normal vector be $\mathbf{n}=$ $\left(x_{n}, y_{n}, z_{n}\right)$, as shown in Fig. 3. From this figure, it is evident that the rotation vector between the planes $\Pi$ and $\Pi^{\prime}$ is the outer product of $\mathbf{n}$ and $\mathbf{d}$ which is the normal vector of $\Pi^{\prime}$, and that the shape of the projected display is that of the cutting plane ( $\Pi$, in this case) of the quadrangular pyramid $\mathbf{Q}$. The algorithm for determining the location, size, and shape of the projected display is summarized as follows:

${ }^{1}$ The errors were evaluated by the distance between the estimated and real positions, and by the angle formed by the estimated and real orientation vectors, respectively. 
1. Find the crossing point $C$ of the extended line from the point $P$ along with the vector $\mathbf{d}$ and the plane $\Pi$. $C$ is the center point of the projected display.

2. Determine the scale $k$ for the projected display on $\Pi^{\prime}$, based on the distance between the point $P$ and the plane $\Pi^{\prime}$ (the length of $\overline{P C}$ in Fig. 3 ).

3. Find the rotation vector between the planes $\Pi$ and $\Pi^{\prime}$ by using $\mathbf{d}$ and $\mathbf{n}$.

4. Determine the shape $S$ of the projected display by the plane $\Pi$ and the quadrangular pyramid $\mathbf{Q}$.

5. Enlarge/reduce the original screen image of the PDA based on the scale $k$, and deform the image to $S$.

Due to the errors as described in 3.2, a projected display of a PDA fluctuates and is not stable, when Hotaru directly uses its estimated position and orientation data. Therefore, Hotaru calculates the PDA's current positions and orientations by averaging over the recent ten consecutive location and orientation data.

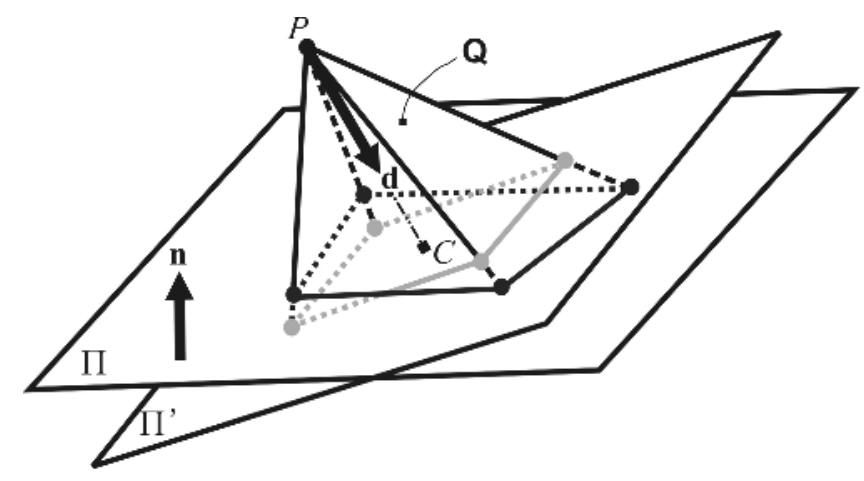

Fig. 3. How Hotaru calculates a projected display of a PDA

\subsection{Detection of Projected Displays}

A video camera attached to a PDA is used for detecting and identifying each of the projected displays and recognizing users' manipulations on them. In order to detect multiple projected displays, a wide-angle lens is mounted on the camera. We first tried to recognize the manipulations by bare fingers as described in [12]. However, the recognition ratio of bare fingers was extremely low without using a special camera such as a thermo-infrared camera 3]. Therefore, in this version of Hotaru, we decided to use fingers augmented with an LED light to increase the recognition ratio (as shown in Fig. 5). The recognition process is summarized as follows:

(1) Extract contours and vertices of projected displays from a captured image through a camera mounted on a user's PDA.

(2) Identify projected displays of individual PDAs (discussed in Section 3.5)

(3) Recognize users' manipulations by fingers (discussed in Section 3.6) 
Fig. 4 shows a process of extracting contours and vertices of a projected display. The system first performs the distortion correction of an image caused due to the wide-angle lens, and applies the Canny edge detector [10] for detecting contours and vertices in the image When Hotaru detects four vertices of individual projected displays, it can successfully recognize the displays. If Hotaru cannot find all the vertices due to occlusion by human hands or an overlap of other projected displays (Fig. $4(\mathrm{a})$ ), it applies the Hough transform (Fig. 4(b)) and estimates the unrecognized vertices in order to determine the regions of projected displays (Fig. 4(c)). Finally, by using the four vertices of the projected display in the camera coordinate and those of the PDA screen in the world coordinate, a transformation matrix between the two coordinates is calculated [10].

(a)

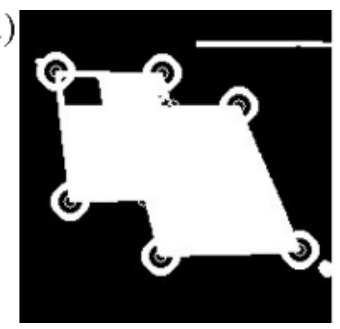

(b)

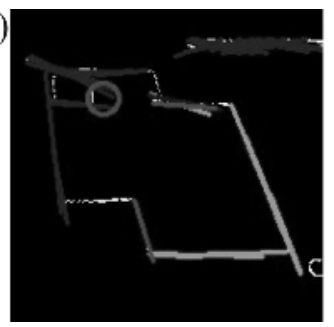

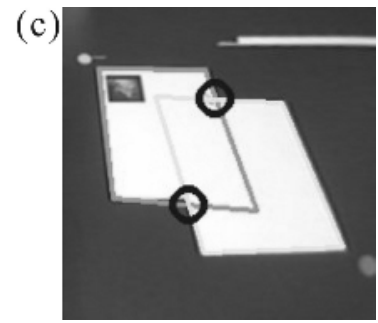

Fig. 4. Extracting a projected display. (a) Three vertices of individual projected displays are determined. (b) The fourth vertex of each projected display is estimated. (c) The region of each projected display is recognized.

\subsection{Identification of Individual Projected Displays}

Although projected displays are detected from an image captured by a camera of PDA, it is not sufficient because which projected displays correspond to individual PDAs has not been determined. By estimating the locations of the projected displays based on the 3D locations and orientations of individual PDAs, it may be possible to find their correspondence. However, if a portable projector mountable on a mobile device has become available, such location and orientation information will be unnecessary. Therefore, the identification of projected displays is conducted by using an image of multiple projected displays captured by a camera mounted on a PDA.

We first tried similarity matching between an elicited projected display and a screen image of each PDA. However, the success ratio of the matching was not stable. It is suggested that screen images of different PDAs were often similar (in some cases, only folder or file icons appeared on their screens). In the current implementation of Hotaru, individual PDAs are given different color markers for their identification. In order to use a screen of a PDA as large as possible, the marker is projected outside of the projected display in Fig. 5 or Fig. 6.

A serious problem of this method is that the number of PDAs to be identified is small (less than ten). To solve the problem, we are now investigating to use a watermark-based method for identifying individual PDAs. 


\subsection{Recognition of Operations by Fingers}

Hotaru allows users to manipulate pictures or file icons on a projected display by using their fingers augmented with an LED light. It elicits a finger-pointing area with a pixel value larger than a specified threshold of brightness. The position of the pointing area on a PDA screen is calculated by using the transformation matrix described in Section 3.4. Hotaru uses the Kalman filter [1] to estimate the next pointing area by using the current and previous pointing areas, when users conduct translation or drag operations explained below. The following is a list of the operations:

- click: when Hotaru recognizes that a user's pointing area has not changed for more than one second, it identifies that the user has conducted a click.

- double click: when Hotaru recognizes that a user's pointing area has not changed for more than two seconds, it identifies that the user has conducted a double click.

- drag: when Hotaru identifies a move of a user's finger after a click, it recognizes the move as a drag.

- release: when Hotaru identifies that a user's finger has stopped for more than one second after a drag, it recognizes the stop as a release.

- cancel: a user can cancel his current operation by moving his finger away from the projected display.

To identify whether a user has really touched a projected display and conducted a click operation with his finger is difficult. Therefore, two-dimensional moves of a user's finger and its non-moving time are used for recognizing his/her operations as described above. The time required for recognizing the operations has been decided through informal user studies during the development of $\mathrm{Ho}_{\mathrm{o}}$ taru. In order to let a user know clearly their current operations, Hotaru provides him/her with different auditory feedback corresponding to each operation.

\section{Intuitive Manipulations on Projected Displays}

Projected displays of mobile devices provide users with new manipulation techniques. Several example techniques are described in the following:

\subsection{Annotation}

When multiple users share a document or a picture on a projected display, they

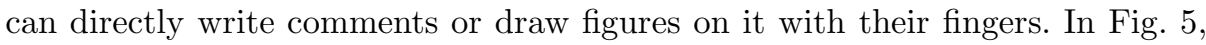
a group of people shares a map on a projected display, and one of them draws lines or marks on it with his finger. Such an annotation is useful in a co-located situation: when one person accesses a web page to show a map by using his mobile device, the other people can easily suggest, recognize or confirm the direction to their destination. 


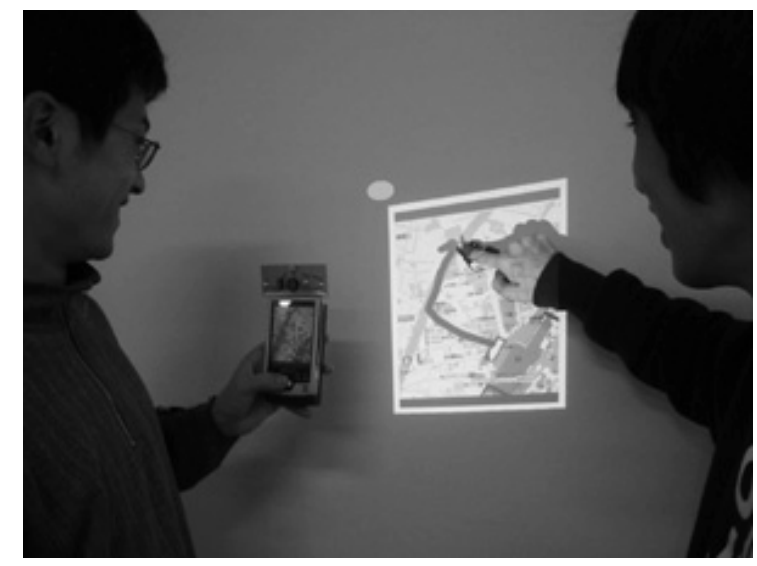

Fig. 5. Annotation to a projected map

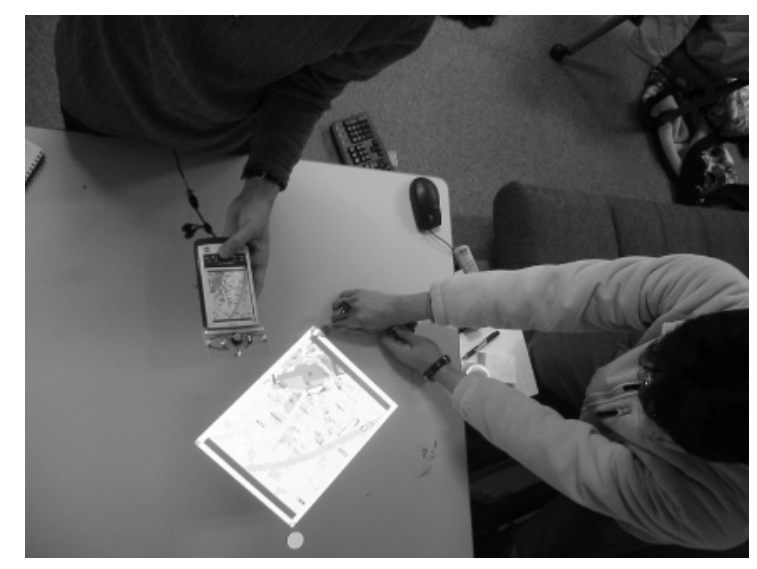

Fig. 6. Rotation of a projected display

\subsection{Rotation}

When an image file in a PDA is projected on a table which multiple people sit around, it is desirable to allow them to rotate the file so that each of them can easily view and understand the content of the image. As shown in Fig. 6, a user can rotate an image about its center point through any angle, by clicking and dragging at the lower right corner of the image.

\subsection{File Transfer by Overlapping Projected Displays}

Fig. 7 shows how users can transfer a file between multiple PDAs. In Fig. 7(a), Displays of two users' PDAs are projected on a table. UserA moves his PDA 
so that its projected display overlaps with that of userB's PDA (Fig. Z(b)), and then drags a image file to be trasferred to the overlapping region of their projected diplays (Fig. Z(c)). The overlapping region is also visualized on the screens of their PDAs. When userA releases the file, a popup window appears on the screen of userB's PDA to confirm if the file transfer is permitted. If userB presses an "ok" button on the popup window, the file is transferred to his PDA as shown in Fig. Z(d). It is also possible for users to write/draw comments on the overlapping region for "carbon-copying" as shown in Fig. Z7(c).

(a)
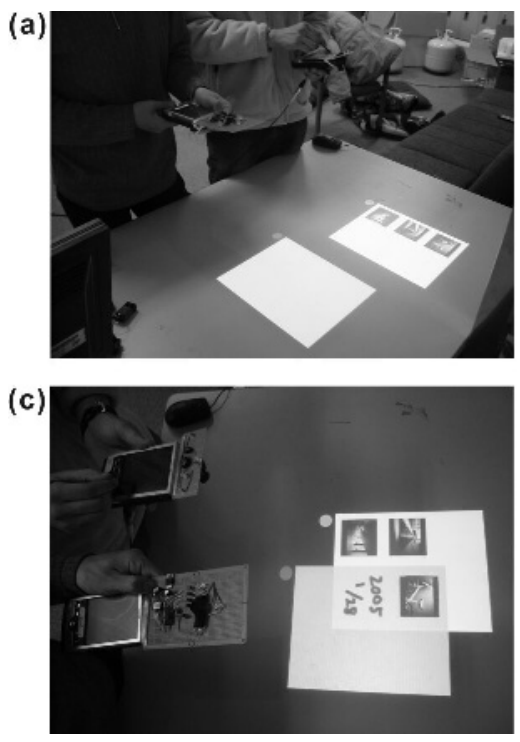

(b)

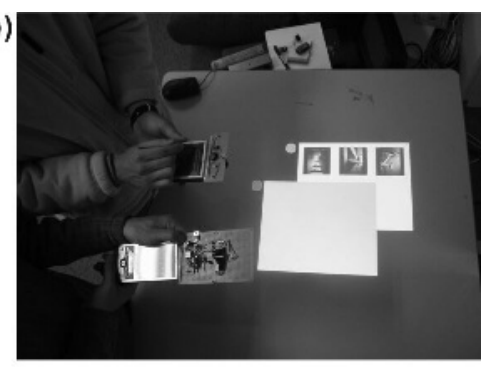

(d)

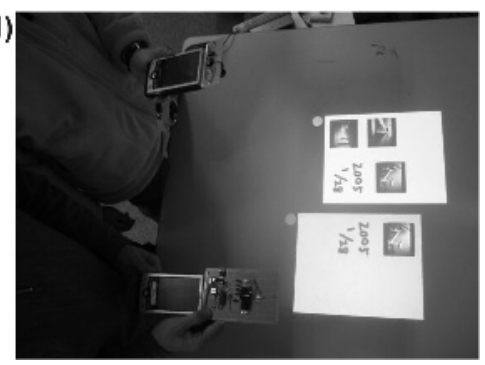

Fig. 7. File transfer between multiple devices by overlapping

\section{User Studies}

Hotaru were evaluated in informal user studies. Twelve subjects formed three pairs and two groups of three, and were asked to conduct the following tasks: annotation and rotation of an image file, and file transfer between multiple PDAs. The tasks lasted about 30 minutes for each pair and group.

During the tasks, users frequently moved their PDAs, and the locations of their projected displays changed accordingly. When the moves of their PDAs were small (such as a slight tilting), Hotaru could almost always recognize their own projected displays correctly. It is suggested that users of Hotaru hold their PDAs steadily rather than move them rapidly, when they want to watch their projected displays or conduct collaborative tasks on them. Therefore, we think that a failure of the recognition due to the move of a PDA is not a serious problem practically. 
Positive (1-4) and negative (5-7) comments received from the subjects are summarized as follows:

1. Projected displays of PDAs were favored users. The users could easily change the positions, shapes, and sizes of the displays as they like, by moving their own PDAs.

2. Hotaru could effectively support multiple people in viewing pictures or documents, because it did not force them to peek at a screen of a PDA over their shoulders.

3. Annotating and rotating a file by fingers were intuitive and useful.

4. Conducting file transfer tasks by overlapping projected displays of PDAs is much more intuitive and easier than other file transfer methods for mobile devices.

5. Slow responses to manipulations by fingers were often irritating.

6. Recognizing users' manipulations failed when a PDA was not held steadily enough (although Hotaru succeeded in recognizing the projected displays).

7. It was desirable to identify who conducted which manipulations, in order for Hotaru to fully support collaborative tasks.

Comments 1-4 indicated that the idea of Hotaru was accepted by the subjects.

The cause of Comment 5 was that the subjects had to stop the moves of their fingers and wait, in order to make click, release, and double click operations recognized by Hotaru. The similar reports are found in [13]. As for Comment 6 , when a subject holding his PDA by one hand tried to touch its projected display with his finger of another hand, the recognition often failed because he unintentionally moved his PDA. Using inertial sensors (e.g. an accelerometer or a gyroscope) or optical flow analyses may be effective to reduce the influence of user's unintentional small moves of his PDA and fix its projected display at a specified location. As for comment 7, several approaches will be possible, for example, using visual or optical tags attached to fingers, in order for Hotaru to identify who has conducted which manipulations.

In the current implementation, Hotaru allows only a sender to conduct file tranfer tasks, that is, a sender first selects a file to be transferred, and then releases it in an overlapping region with a projected display of another PDA. All the subjects requested that Hotaru should also allows a receiver to conduct file transfer tasks by selecting other users' file that appear on the receiver's PDA screen. We will plan to improve and extend functions of Hotaru by examining the issues raised through the user studies.

\section{Conclusions}

This paper described a system called Hotaru and possible intuitive manipulation techniques. The design and implementation of Hotaru by using currently available technologies were discussed. Informal user studies for evaluating $\mathrm{Ho}$ taru proved that it would effectively support collaborative tasks in co-located 
situations. The studies also clarified several problems to be solved and investigated. In our future work, we will conduct more intensive user studies, improve the performance of Hotaru, and explore its possibilities of new applications.

\section{References}

1. Ayatsuka, Y., et al: HyperPalette: a Hybrid Computing Environment for Small Computing Device, Proceedings of ACM CHI2000, pp.133-134 (2000).

2. Greenberg, S., et al: PDAs and Shared Public Displays: Making Personal Information Public and Public Information Personal. Personal Technologies, Vol.3, No.1, pp.54-64 (1999).

3. Koike, H., et al.: Integrating Paper and Digital Information on EnhancedDesk: A Method for Real-Time Finger Tracking on Augmented Desk System, ACM Transactions on Computer Human Interaction, Vol.8, No.4, pp.307-322 (2001).

4. Japanese Ministry of Internal Affairs and Communications : Information and Communication in Japan 2004 (available at http://www.johotsushintokei. soumu.go.jp/whitepaper/eng/WP2004/2004-index.html (2004).

5. New York Times:For Your Viewing Pleasure, a Projector in Your Pocket (2004) (available at http://www.nytimes.com/2004/11/04/technology/circuits/ 04next.html).

6. Pingali, G., et al. (2003): Steerable Computing Interfaces for Pervasive Spaces, in Proccedings of IEEE PerCom2003, pp.315-322 (2003).

7. Raskar, R., et al. (2003): iLamps: Geometrically Aware and Self-Configurating, ACM Transactions on Graphics, Vol.22, No.3, pp.809-818 (2003).

8. Rekimoto, J., Saito, M.:Augmented Surfaces: A spatially Continuous Work Space for Hybrid Computing Environments, Proceedings of ACM CHI99, pp.378-385 (1999).

9. Roeber, H., et al.: Typing in Thin Air: the Canesta Projection Keyboard - A New Method of Interaction with Electronic Devices, Proceedings of ACM CHI2003, pp.712-713 (2003).

10. Trucco, E., Verri, A.: Introductory Techniques for 3-D Computer Vision, Prentice Hall (1998).

11. Welch, G., Bishop, G.: An Introduction to the Kalman Filter, SIGGRAPH 2001 Tutorial (2001) (available at http://www.cs.unc.edu/ tracker/ media/pdf/SIGGRAPH2001_CoursePack_08.pdf).

12. Wellner, P.: Interacting with Paper on the DigitalDesk, Communications of the $A C M$, Vol.36, No.7, pp.87-96 (1993).

13. Zhang, Z., et al.: Visual Panel: Virtual Mouse, Keyboard and 3D Controller with an Ordinary Piece of Paper, Proceedings of Perceptual User Interface, pp.1-8 (2001). 